\title{
Anxiety and palpitations in patients with cardiac implantable electronic devices (CIEDs) and the added value of remote monitoring (RM) in the COVID-19 pandemic: an Italian single-centre experience
}

\author{
TERESA STRISCIUGLIO ${ }^{1}$, Giuseppe Ammirati ${ }^{1}$, Livio Imparato ${ }^{2}$, Valerio Pergola ${ }^{1}$, Lucio \\ Addeo $^{1}$, Aniello Viggiano ${ }^{1}$, and Antonio Rapacciuolo ${ }^{3}$ \\ ${ }^{1}$ University of Naples Federico II \\ ${ }^{2}$ Federico II University Hospital \\ ${ }^{3}$ Federico II University of Naples
}

June 5, 2020

\begin{abstract}
Background. Mood disorders are common in patients with cardiac implantable electronic devices (CIEDs). The lockdown related to COVID-19 pandemic may have further undermined their emotional well being exacerbating physical symptoms such as palpitations. We investigated the occurrence of anxiety and palpitations in CIEDs patients during the pandemic. In those with remote monitoring (RM) available, we evaluated whether these symptoms were associated with arrhythmic events. Methods and Results. The generalized anxiety disorder (GAD) 7-items questionnaire and the frequency of palpitations were assessed by telephonic interview in CIEDs patients Overall 152 CIEDs patients agreed to be interviewed (119 males, age $69 \pm 14$ years). The RM was available in 54/152 (36\%). Thirtyseven (24\%) and $43(28 \%)$ patients experienced anxiety and palpitations respectively, and 23 (15\%) patients experienced both. Women were more likely to have symptoms than men (50\% vs. $20 \%$; $\mathrm{p}<0.01$ for anxiety and $53 \%$ vs. $24 \%$; $<<0.01$ for palpitations). Among patients with palpitations the RM was available in $17 / 43(40 \%)$ and revealed the following arrhythmias in 7/17 (41\%) patients: 8 non-sustained ventricular tachycardias (NSVT), 2 supra-ventricular tachycardias (SVT), 4 sustained ventricular tachycardias (VT). The RM enabled to detect in 6 patients a device malfunctioning: in 2 increased impedance in the shock lead, in 3 increased capture threshold for the LV lead and in 1 the battery reached the end of life Conclusion. Patients with CIEDs during the COVID19 outbreak were likely to experience anxiety and palpitations and the RM has been pivotal to ascertain the occurrence of arrhythmic events and device malfunctioning
\end{abstract}

\section{Introduction}

The outbreak of the pandemic caused by coronavirus disease 2019 (COVID 19) led the hospitals to "reshape" themselves in order to dedicate the majority of the efforts, in terms of human and economic assets, in the management of infected patients.

These changes of course involved also the Cardiac Electrophysiology (EP) Departments and in order to counteract the contagion the face-to-face EP visits, unless urgent, have been avoided and tele-medicine and remote monitoring (RM) of patients with cardiac implantable electronic devices (CIEDs) have been encouraged to reduce the exposure.

Furthermore, the COVID-19 pandemic has forced the governments of the most heavily affected countries to adopt a complete lockdown policy for about 2 months with dramatic consequences on the mental health of individuals (1-4), indeed mood disorders and suicides increased. 
As depression and anxiety are common in CIEDs patients, especially in those having an ICD (6-7), these challenging times may undermine their emotional well being exacerbating physical symptoms either true or "psychogenic".

In the current study we investigated the occurrence of anxiety and palpitations among CIEDs patients during the pandemic, and in those for which the RM was available we investigated whether these symptoms were associated with arrhythmic events or device malfunctioning.

\section{Methods}

\section{Study population}

In our centre, the Department of Cardiology of the University of Naples Federico II (IT), CIED patients are followed-up with outpatient visits every 6 - and 12 - months and for about $1 / 3$ of these the RM it is also available.

\section{Events collection by RM}

Since the start of the lockdown (i.e. March 2020) we were able to check the functioning of the device and the occurrence of arrhythmic events only in patients for which the RM was available.

Two EP technicians performed a remote daily check of these patients and looked for any pre-specified alerts related to device functioning or to clinical events.

Thereafter, the alerts were evaluated by 2 electrophysiologists in order to discard the not relevant ones.

Assessment of anxiety and palpitations

Four different physicians (GA, VP, LI, LA) contacted by telephone and interviewed all patients whose control visit was scheduled in the months of the lockdown period (March-April) and due to the COVID-19 was cancelled. The presence of anxiety during the lockdown period was assessed by the (General Anxiety Disorder) GAD-7 questionnaire (5). The latter comprises 7 questions that investigate the occurrence of uncomfortable feelings related to anxiety, and for each answer a score from 0 to 3 is attributed, based on the frequency of these feelings $(0=$ never; $1=$ several days; $2=$ more than half of the days; $3=$ nearly every day; $\min -\max =0-21$ ). A score [?]5 indicates mild anxiety, a score [?]10 indicates moderate anxiety and [?]15 indicates severe anxiety. Likewise patients were also asked to describe whether they felt palpitations during the index period and their frequency $(0=$ never; $1=$ once per month; $2=$ once per week; $3=$ nearly every day; min-max $=0-3$ ) (Figure 1). All patients gave the informed consent to data collection. The study is in agreement with the Declaration of Helsinki e was approved by the local ethical committee.

\section{Statistical analysis}

Descriptive statistics are reported as means \pm standard deviations for normally distributed continuous variables or medians with 25 th to 75 th percentiles in the case of skewed distribution. Normality of distribution was tested by means of the nonparametric Kolmogorov-Smirnov test. Differences between mean data were compared by means of a t-test for Gaussian variables, and the F-test was used to check the hypothesis of equality of variance. The Mann-Whitney non-parametric test was used to compare non-Gaussian variables. The difference in proportions was tested by applying the $\chi^{2}$ analysis or the Fisher's exact test, when appropriate. Pearson's correlation was performed to assess the correlation between anxiety and palpitations scores. All statistical analyses were performed by means of SPSS 25.0 (IBM. Armonk. New York. USA).

\section{Results}

Study population and prevalence of anxiety and palpitations

Between March and April 2020, 169 CIEDs control visits were scheduled in our outpatient clinic and all of them had to be cancelled. All patients whose visit was cancelled were contacted by telephone, and 152 of them agreed to answer to the questionnaire whereas 2 had died of non-cardiac death. One hundred and nineteen patients $(78 \%)$ were males with a mean age of $69 \pm 14$ years. Sixty-seven patients had an implantable 
cardiac defibrillator (ICD), 37 had a cardiac resynchronization device (CRT), 40 had a pacemaker (PMK) and 8 had an implantable loop recorder (ILR). The RM was available for 54 out of $152(36 \%)$ patients.

Overall, $37(24 \%)$ and $43(28 \%)$ patients experienced anxiety (at least mild [?] 5) and palpitations respectively, and $23(15 \%)$ patients experienced both (Figure 2). No differences were found in the occurrence of anxiety and palpitations between patients with an ICD/CRTD and those with a CRTP, PMK or ILR (Figure 3 , panel A).

Women were more likely to have these symptoms than men (50\% vs. $20 \%$; $<<0.01$ for anxiety and $53 \%$ vs. $24 \% ; \mathrm{p}<0.01$ for palpitations) (Figure 3, panel B). The mean GAD7 score was 5+-4.5, however the score was significantly higher in women $(7.5+-5.5$ vs. $4.1+-3.5 ; \mathrm{p}<0.01)$. Furthermore, significantly more women than men reported to have palpitations at least once per week or nearly every day (palpitation score $2 / 3$ ) ( $38 \%$ vs $17 \%, \mathrm{p}=0.02)$.

\section{Correlation between anxiety and palpitations}

We found a significant moderate correlation between the prevalence of anxiety and the occurrence of palpitations $(\mathrm{r}=0.45)$. In particular, we found that when dividing the overall population in tertiles of anxiety (GAD7 score), the mean palpitations score was higher in the highest tertile $(\mathrm{p}<0.01)$ (Figure 4$)$.

Arrhythmic events detected by remote monitoring in patients with and without palpitations

Among the 43 patients who experienced palpitations, the RM was available in 17 patients (40\%), thus in these we were able to verify the occurrence of arrhythmic events. In 7 out of $17(41 \%)$ patients the palpitations were really associated with arrhythmic events: 4 patients experienced a single episode of non sustained ventricular tachycardia (NSVT), 1 patient experienced an episode of supraventricular tachycardia (SVT) and an episode of NSVT, 1 patient experienced a single episode of SVT and finally 1 patient experienced 3 NSVT and 4 sustained ventricular tachycardias (VTs) for which he received shock (Table 1).

For those 37 patients for whom the RM was available however that didn't report the occurrence of palpitations, we identified the occurrence of arrhythmic events in $4(11 \%)$ of them: in 2 a new onset of atrial fibrillation (AF) and in the other 2 patients a single episode of fast VT (>210bpm) treated with ATP and shock (Table 1).

Patients with new onset of AF were then contacted to start an adequate anti-coagulation therapy according to the CHA2DS2VASc score and in the absence of contraindications, whereas patients with recurrent SVT were scheduled for a 24-hours Holter-ECG. In patients with NSTV and TVs the anti-arrhythmic therapy was implemented according to the electrophysiologist's discretion.

\section{Cases of device malfunctioning detected by remote monitoring}

By mean of the RM the alerts related to device malfunctioning were detected in 6 patients.

In particular, in 2 ICD patients the alerts revealed increased impedance in the shock lead, and as this was considered an urgent situation, patients were asked to come to the hospital. In the cathlab, under deep sedation we induced an episode of fast $\mathrm{VT} / \mathrm{VF}$ and we ascertained the correct functioning of the shock lead. In other 3 CRT patients who reported the occurrence of fatigue and dyspnoea the RM revealed that the rate of BiV pacing was very low as the threshold of capture of the left ventricular lead was too high with loss of capture, therefore, again this was considered an urgent situation, and patients were asked to come to the hospital and we changed the pacing vector to reach an increased BiV pacing. Finally, in 1 patient the generator of the PMK reached the end of life (EOL) and the patient was scheduled for an urgent replacement of the battery (Table 2).

\section{Discussion}

Main findings 
The current study demonstrates that in the COVID-19 pandemic 1) CIEDs patients were likely to have anxiety and palpitations, and in particular women more than men 2) the frequency of palpitations in CIEDs patients was related to their anxiety status 3 ) the RM enabled to verify the true arrhythmic events associated with palpitations and to properly treat these patients when needed, whereas in CIEDs patients not followed by RM the incidence of real arrhythmic events could not be ascertained 4) the RM enabled to identify patients with device malfunctioning and to properly manage them.

The impact of COVID-19 pandemics and of the lockdown on the Italian population

The complete lockdown adopted by the governments dramatically affected the population. Beyond having worries about their own health and that one of their relatives, people had to worry about the financial consequences of the working activities stop $(1,2)$. Recent data also demonstrate that in Italy patients were afraid of getting infected by COVID-19 in the hospitals, thus even in case of cardiovascular symptoms, such as chest pain, they didn't go to the emergency departments. This wrong behaviour, reflected by the incredibly lower number of accesses to the emergency departments for myocardial infarction and its increased mortality, was observed all-over the country and especially in highly populous regions as Campania region $(3,4)$.

There is a tight relationship between mood disorders and cardiovascular diseases and it is well established that depression and its related symptoms may influence the outcomes of patients with cardiovascular disease (5). Therefore in this category of patients the emotional wellbeing is pivotal.

Previous studies reported that depression and anxiety are present in nearly half of the patients with ICD (6-8). These challenging times may have severely impacted the mental health of patients with CIEDs, in particular of those who have seen their control outpatient visit cancelled. We hereby demonstrated that nearly 1 out of 4 patient felt anxious and experienced palpitations at least once during the months of the lockdown, and especially women were more likely to feel these symptoms. Furthermore, we found that anxiety and palpitations were tightly related.

The benefits of remote monitoring of CIEDs

Outpatient visits for device interrogation are usually scheduled every 6-12 months, however the RM is a valid option to reduce them.

More than a decade ago Heidbuchel et al. (9) demonstrated that most of the arrhythmia- and/or devicerelated events in ICD patients are found during the unscheduled visits, and that the ICD remote monitoring in addition to a clinical follow-up performed by the general practitioner may potentially diagnose $>99.5 \%$ of arrhythmia- or device-related problems. Since then the CIEDs companies have implemented their RM systems and many studies provided evidence of the important contributions of this new technology. In particular the CONNECT and the TRUST trial (10-11) proved a significant reduction in the total number of in-office visits, with consequent huge cost savings for the health systems. Moreover, the TRUST trial demonstrated also significant benefits for the patients, as the silent or symptomatic arrhythmic/ device problems were detected about 30 days earlier with RM than with programmed in-office interrogations (11).

In the current study we demonstrated the added value of RM in the COVID-19 pandemic, as in such "extraordinary" situation it represented the only way to detect relevant events in CIEDs population especially in those patients who reported to have symptoms such palpitations. In particular, we identified 18 arrhythmic events in 11 patients (7 symptomatic and 4 asymptomatic): 8(44\%) NSVT, 2(11\%) SVT, 2(11\%) new onset $\mathrm{AF}, 6(33 \%) \mathrm{TV}$.

Furthermore, the following scenarios of device malfunctioning were detected via the RM: increase in the impedance of the shock lead, EOL of the PMK battery, increase of the threshold of LV lead capture.

\section{Clinical implications}

The population of CIEDs patients is rapidly increasing all-over the world, and as the devices require regular technical checks, cardiologists are increasingly overworked. The RM was demonstrated to reduce the number 
of in-office visits, however some doubts were raised about the patient perception of this new technology, as in this way the face-to-face contact with their caregivers would be lost. Actually it has been demonstrated that patients showed a high level of acceptance and satisfaction for this new aspect of the healthcare organization and up to $92 \%$ of patients claimed to receive a sense of security by having at home the transmitter (12). The COVID-19 emergency highlighted the pivotal role of RM, and especially the need of extending this type of monitoring to the vast majority of the CIEDs patients. So far, however only in few countries the physicians are reimbursed for RM, and this represents the main obstacle for the widespread of this technology. In Italy the RM is not reimbursed, and especially in areas such as Campania region, with limited economic resources intended for healthcare, this technology would be very helpful to cut the costs of unnecessary outpatient visits. Furthermore, the occurrence of this pandemic may represent an incentive for the governments to implement and reimburse the RM as this represent the only way to dramatically reduce or even abolish the risk of contagion.

\section{Limitations}

This is a single-centre study, thus the number of patients included is limited. Furthermore, In Italy the RM is not reimbursed therefore only $36 \%$ of patients were followed with this technology. As a consequence we were not able to detect relevant arrhythmias or device malfunctioning in the other patients without RM.

\section{Conclusions}

Patients with CIEDs, especially women, during the COVID19 outbreak were likely to experience anxiety and palpitations. In the lockdown period the RM has been pivotal as it represented the only way to ascertain the occurrence of arrhythmic events and device malfunctioning and to properly manage them.

\section{References}

1. Kawohl W, Nordt C. COVID-19, unemployment, and suicide. Lancet Psychiatry. 2020;7(5):389-390. doi: 10.1016/S2215-0366(20)30141-3.

2. Pfefferbaum B, North CS. Mental Health and the Covid-19 Pandemic. N Engl J Med. 2020 Apr 13. doi: 10.1056/NEJMp2008017.

3. De Rosa S, Spaccarotella C, Basso C, Calabro MP, Curcio A, Perrone Filardi P, Mancone M, Mercuro G, Muscoli S, Nodari S, Pedrinelli R, Sinagra G, Indolfi C, Societa Italiana di Cardiologia and the CCU Academy investigators group. Reduction of hospitalizations for myocardial infarction in Italy in the COVID-19 era. Eur Heart J. 2020 May 15. pii: ehaa409. doi: 10.1093/eurheartj/ehaa409.

4. Piccolo R, Bruzzese D, Mauro C, Aloia A, Baldi C , Boccalatte M, Bottiglieri G, Briguori C, Caiazzo G, Calabro P , Cappelli-Bigazzi M, De Simone C2, Di Lorenzo E, Golino P, Monda V, Perrotta R, Quaranta G, Russolillo E, Scherillo M, Tesorio T, Tuccillo B, Valva G, Villari B, Tarantini G , Varricchio A, Esposito G. Population Trends in Rates of Percutaneous Coronary Revascularization for Acute Coronary Syndromes Associated with the COVID-19 Outbreak. Circulation. 2020 Apr 30. doi: 10.1161/CIRCULATIONAHA.120.047457.

5. Lowe B, Decker O, Muller S. Validation and Standardization of the Generalized Anxiety Disorder Screener (GAD-7) in the General Population. Med. Care 2008 Mar;46(3):266-74.

6. Musselman DL, Evans DL, Nemeroff CB. The Relationship of Depression to Cardiovascular Disease. Arch Gen Psychiatry. 1998;55(7):580-592. doi:10.1001/archpsyc.55.7.580

7. Lang S, Becker R, Wilke S, Hartmann M, Herzog W, LoweB. Anxiety disorders in patients with implantable cardioverter defibrillators: frequency, course, predictors, and patients' requests for treatment. Pacing Clin Electrophysiol. 2014 Jan;37(1):35-47. doi: 10.1111/pace.12276.

8. Bilge AK, Ozben B, Demircan S, Cinar M, Yilmaz E, Adalet K. Depression and anxiety status of patients with implantable cardioverter defibrillator and precipitating factors. Pacing Clin Electrophysiol. 2006;29(6):619-26.

9. Heidbuchel H, Lioen P, Foulon S, Huybrechts W, Ector J, Willems R, Ector H. Potential role of remote monitoring for scheduled and unscheduled evaluations of patients with an implantable defibrillator. Europace 2008;10:351-7. 
10. Crossley G, Boyle A, Vitense H, Sherfesee L, Mead RH. The Clinical Evaluation of Remote Notification to Reduce Time to Clinical Decision (CONNECT) Trial. Am Heart J. 2008;156:840-6.

11. Varma N, Epstein A, Irimpen A, Schweikert R, Love C. The lumos-T safely reduces routine efficacy and safety of automatic remote monitoring for implantable cardioverter-defibrillator follow-up. The Lumos-T Safely Reduces Routine Office Device Follow-Up (TRUST) Trial. Circulation 2010;122:32532.

12. Ricci RP, Morichelli L, Quarta L, Sassi A, Porfili A, Laudadio MT, Gargaro A, Santini M. Long-term patient acceptance of and satisfaction with implanted device remote monitoring. Europace 2010; 12: 674-679.

13. Mairesse GH, Braunschweig F, Klersy K, Cowie MR, Leyva F. Implementation and Reimbursement of Remote Monitoring for Cardiac Implantable Electronic Devices in Europe: A Survey From the Health Economics Committee of the European Heart Rhythm Association. Europace 2015; 17: 814-818

Figure Lengend

Figure 1. Questionnaires for the assessment of anxiety and palpitations

Figure 2. Prevalence of anxiety and palpitations in CIED patients

Figure 3. Prevalence of anxiety and palpitations according to the device type (upper graph) and to the gender (lower graph)

Figure 4. Mean palpitation score per tertile of GAD7 score

Figure 1

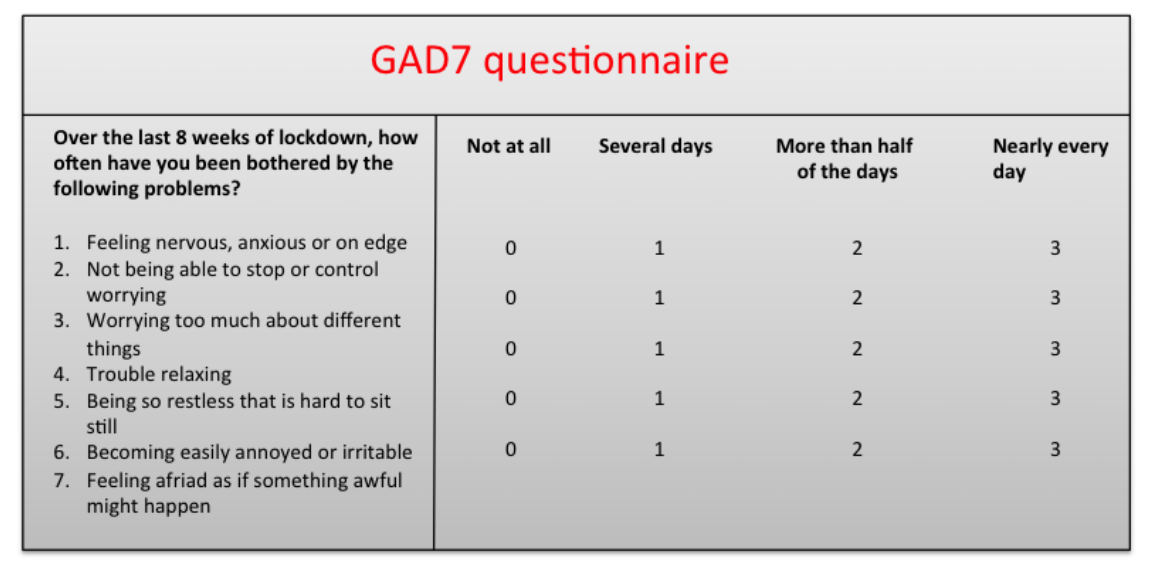

\begin{tabular}{|l|cccc|}
\hline \multicolumn{4}{|c|}{ Palpitations questionnaire } \\
\hline $\begin{array}{l}\text { Over the last } 8 \text { weeks of lockdown, } \\
\text { how often have you experienced } \\
\text { episodes of palpitations? }\end{array}$ & Never & Once per month & Once per week & $\begin{array}{l}\text { Nearly every } \\
\text { day }\end{array}$ \\
\hline
\end{tabular}

Figure 2 


\section{CIEDs patients were contacted}

by telephone

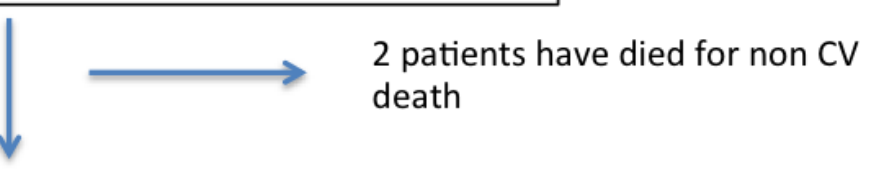

\section{2 accepted to be interviewed}

GAD7 and palpitation

questionnaires

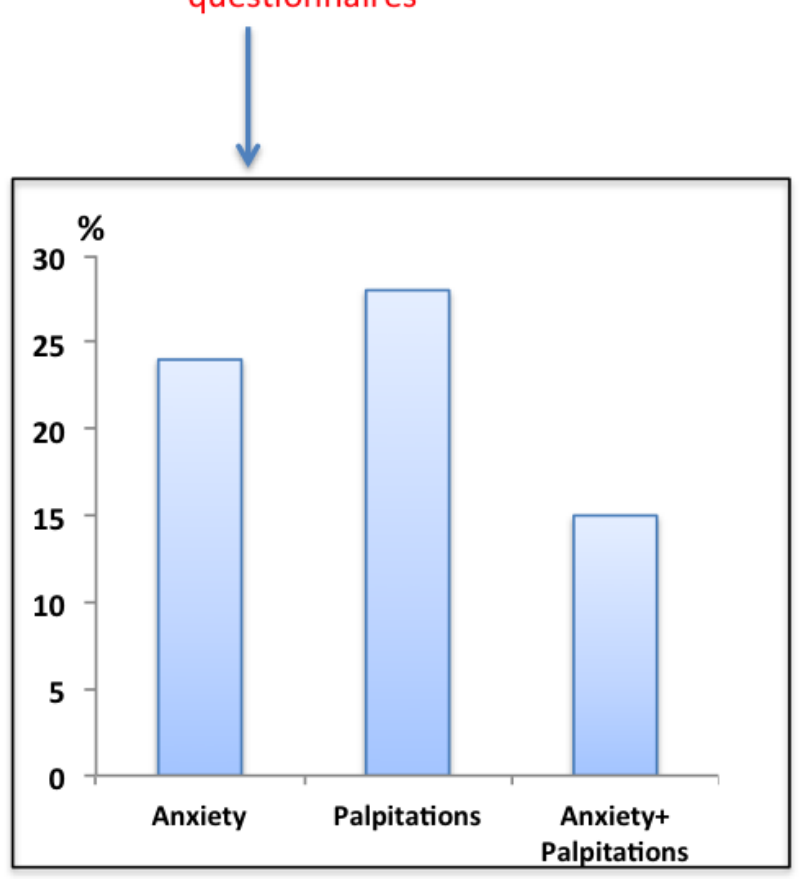

Figure 3 
A

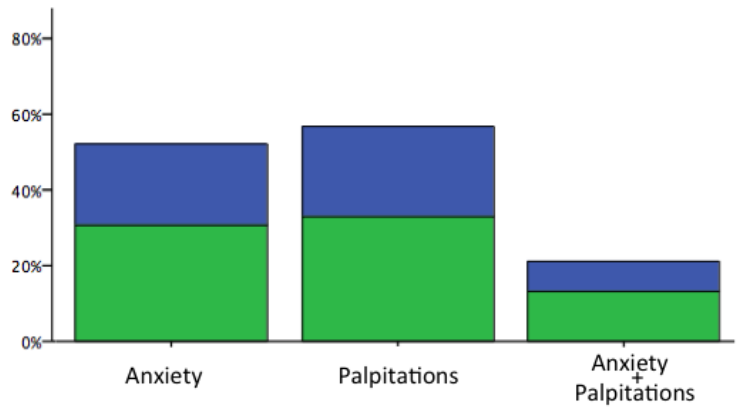

B

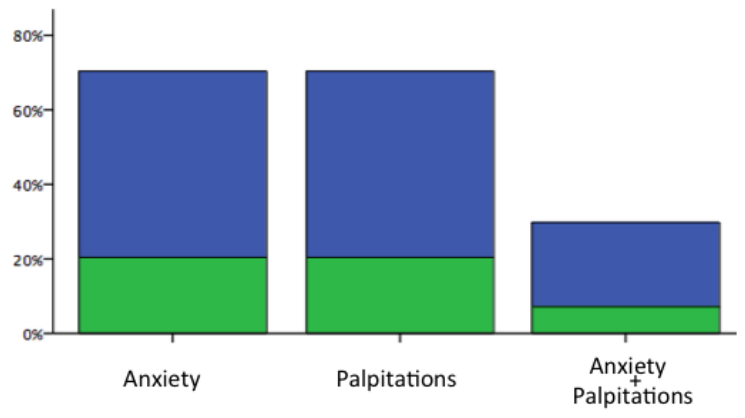

PMK, CRTP, ILR

Figure 4

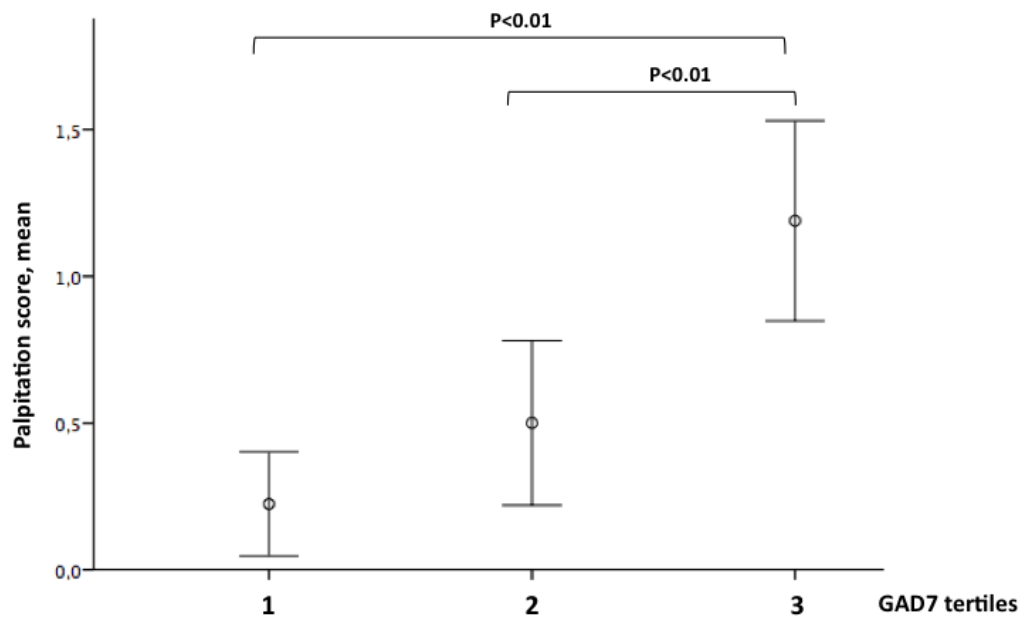

Table 1. Cases of arrhythmic events detected by remote monitoring of CIEDs 


\begin{tabular}{|c|c|c|c|c|c|}
\hline $\begin{array}{l}\text { Patients with } \\
\text { palpitations }\end{array}$ & Gender & Age & Device & Brand & Arrhythmias \\
\hline C.G. & F & 61 & ICD & BOSTON & $\begin{array}{c}\text { 1 SVT } \\
\text { 1 NSVT }\end{array}$ \\
\hline D.A. & M & 46 & ICD & BOSTON & 1NSVT \\
\hline F.F. & M & 62 & ICD & BOSTON & 1NSVT \\
\hline A.F. & M & 56 & ICD & BIOTRONIK & 1NSVT \\
\hline C.F. & M & 85 & CRTD & BOSTON & 1 SVT \\
\hline A.M. & F & 63 & PMK & BIOTRONIK & 1NSVT \\
\hline R.G. & M & 55 & CRTD & BIOTRONIK & $\begin{array}{c}\text { 3NSVT } \\
4 \text { TV }\end{array}$ \\
\hline $\begin{array}{l}\text { Patients with no } \\
\text { palpitations }\end{array}$ & & & & & \\
\hline G.U. & M & 83 & ICD & BIOTRONIK & 1 TV \\
\hline R.A. & M & 58 & ICD & ST.JUDE & 1NSVT \\
\hline M.V. & M & 73 & PMK & BIOTRONIK & AF \\
\hline A.R. & M & 66 & ICD & BIOTRONIK & AF \\
\hline
\end{tabular}

ICD: implantable cardiac defibrillator; CRTD: cardiac resynchronization therapy-defibrillator; PMK: pacemaker; SVT: supra-ventricular tachycardia; NSVT: non sustained ventricular tachycardia; VT: ventricular tachycardia

Table 2. Cases of device malfunctioning detected by remote monitoring of CIEDs

\begin{tabular}{|c|c|c|c|c|c|}
\hline $\begin{array}{l}\text { Patients with } \\
\text { device-related } \\
\text { alerts }\end{array}$ & Gender & Age & Device & Brand & Type of alert \\
\hline A.V. & M & 84 & CRTD & MEDTRONIC & $\begin{array}{c}\text { Loss of capture } \\
\text { of LV lead }\end{array}$ \\
\hline T.S. & M & 77 & CRTD & ST.JUDE & $\begin{array}{c}\text { Loss of capture } \\
\text { of LV lead }\end{array}$ \\
\hline D.S. & F & 67 & ICD & BOSTON & $\begin{array}{c}\text { Shock lead } \\
\text { impedance out } \\
\text { of range }\end{array}$ \\
\hline B.A. & F & 68 & ICD & BIOTRONIK & $\begin{array}{c}\text { Shock lead } \\
\text { impedance out } \\
\text { of range }\end{array}$ \\
\hline N.S. & M & 83 & CRTD & BIOTRONIK & $\begin{array}{c}\text { Loss of capture } \\
\text { of LV lead }\end{array}$ \\
\hline C.A. & M & 80 & PMK & SORIN & Battery EOL \\
\hline
\end{tabular}

ICD: implantable cardiac defibrillator; CRTD: cardiac resynchronization therapy-defibrillator; PMK: pacemaker; LV: left ventricle; EOL: end of life 